\title{
Efficient virtual garment fit evaluation infrastructure based on synthetic avatar target customer groups for MtM application
}

\author{
Michael Ernst ${ }^{1}$, Antje Christophersen ${ }^{1}$, Monika Böhm¹, Ulrich Botzenhardt² \\ ${ }^{1}$ Hochschule Niederrhein - University of Applied Sciences, Virtual Lab, Mönchengladbach, Germany, \\ michael.ernst@hs-niederrhein.de, antje.christophersen@hs-niederrhein.de, monika.boehm@hs-niederrhein.de \\ ${ }^{2}$ Avalution GmbH, Kaiserslautern, Germany, ulrich.botzenhardt@avalution.de
}

\section{INFO}

CDAPT, ISSN 2701-939X

Peer reviewed article 2020, Vol. 1, No. 2, pp. 160-169

DOI 10.25367/cdatp.2020.1.p160-169

Received: 30 June 2020

Accepted: 13 December 2020

Available online: 22 December 2020

\author{
Keywords \\ Mass customization, \\ test population, \\ target group, \\ sizing and fit, \\ fit control, \\ 3D simulation, \\ virtual product development
}

\begin{abstract}
Customization becomes more and more popular and influences the product development process in apparel industry. In addition to individualized products, the fit of garments is very important for the customization. Numerous tools are used to take the right measurements, to transport individual posture information and to implement these data correctly into a product pattern based on a predefined construction system. Unfortunately, in most cases the mass customization process takes place without a fitting session. Usually fit and design will be checked in the last process step, when the product is already manufactured. Virtual product development is a powerful tool to change this process getting an early fit and design check. By using a test population representing the target group, it is possible to check the sizing and to screen the fit of a product on individual bodies and postures in a short time. In a joint project between the Virtual Lab of Niederrhein University of Applied Sciences and Avalution $\mathrm{GmbH}$, a practical approach for the implementation of a fitting session to a mass customization product development process was developed. The entire process has a three-level structure: First, the avatar population is built up using garment specific body measurements. Connected to a $3 D$ simulation program, an automatic process of determining the madeto-measure (MtM) values, carrying out the MtM grading and the fitting on the selected avatar are initiated. In a special application, the digital try-ons are finally output as images in different physical aspects for evaluation.
\end{abstract}

(C) 2020 The authors. Published by CDAPT.

This is an open access article under the CC BY-NC-ND license https://creativecommons.org/licenses/ peer-review under responsibility of the scientific committee of the CDAPT. 


\section{Introduction}

The shape of the human body is individual. A wide range of body types and dimensions can be observed. Pattern systems that aim for a good fit, even more MtM systems that promise a good individual fit for a really wide range of potential customers are critical, but in any case, expensive to evaluate.

This typically leads to two extreme situations during operation. Conservatively speaking, some systems remain completely unchanged during operation (never change a running system) and therefore potentially have a suboptimal success on the market. Other systems are constantly changed during production operation, with a correspondingly high risk that these changes are only focused on the last short-term adjustments. Although obviously introduced with the best intentions, these changes can have undesirable negative side effects or even destabilize the entire system.

In order to prevent such a situation, it is therefore essential to validate and optimize such complex systems prior to commissioning. If changes are required and the system is already productive, it is important to have a tool that somehow broadens the perspective, shows side effects of changes and thus helps to avoid deteriorating the overall performance of the system.

It is therefore essential to have a simulation system available that reduces these risks by virtually validating the system or its behavior on a variety of body types [1,2] (given by so-called "avatar test populations"), specifically designed for a single target group or covering a wide range of customers, in order to improve size sets in the apparel industry.

\section{Technological Approach}

The fit is the most critical part of a successful "made to measure" process in the garment industry [1].

Frequently, the individual fit is determined by means of a second grading, the so-called MtM grading. MtM grading means an additional grading process based on the standard grading. First the reference size is assigned, then the MtM grading is used.

The structure of the MtM grading is very complex, because every customer has different values and especially different combinations of body measurements.

The iMtM process developed in this project is based on the use of $3 D$ technologies and thus offers considerable advantages over the established 2D MtM process. An avatar population representing the MtM customers, garment simulations for fit control and last but not least the automation within these processes characterise the strength of the 3D technologies and thus the advantages of the iMtM process.

An overview of the technological approach of the so-called iMtM process is shown in figure 1. The iMtM process chain starts with the generation of avatar test populations, which are connected to a garment simulation system via an innovative batch simulator, resulting in a viewer that offers a new approach to fit assessment. The fit is the most critical part of a successful "tailor-made" process in the garment industry [1].

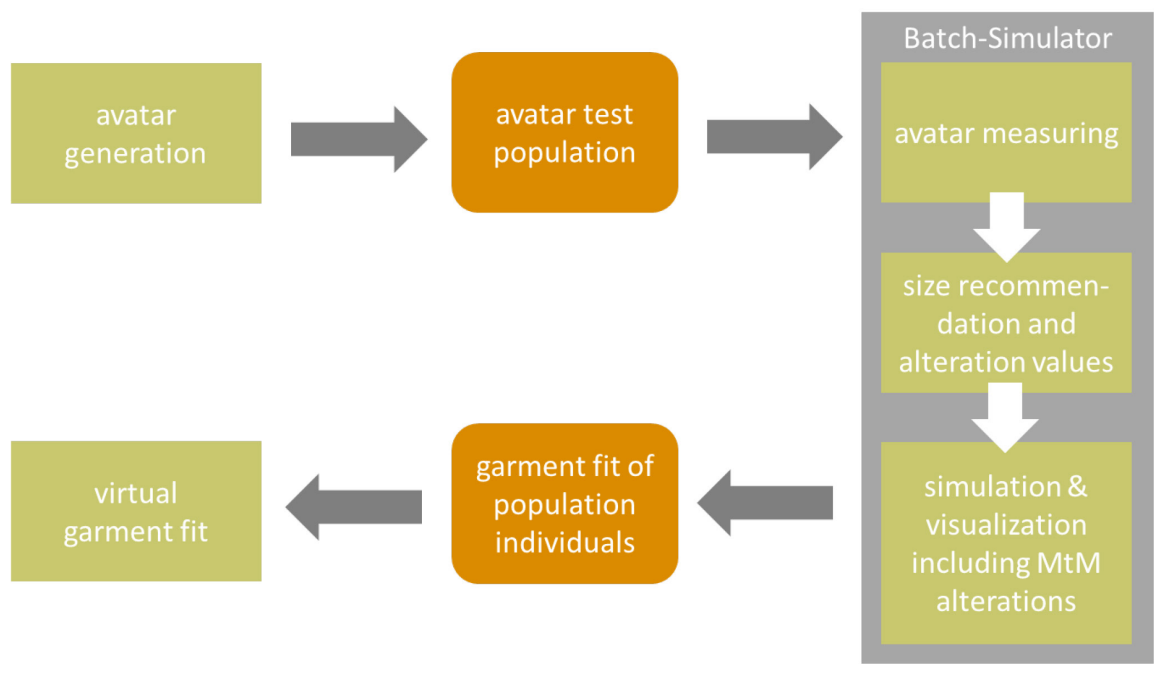




\subsection{Definition and synthesis of the target group}

The technology presented in this paper is primarily based on the so-called AvatarStudio technology. This technology currently drives the avatar generator "AvatarTool" in Vidya [3]. In the "Vidya" implementation, it enables the creation of 3D avatars using typical standard body dimensions. The technology "3D-Side" is based on a principal component analysis (PCA) approach using all 3D scan material (>>10k scans) collected within the measurement series Size Germany [4]. It goes beyond the aim of this paper to present the working principles of PCA and its theory in detail, but it is important to understand that PCA acts in a certain way like a learning method, causing extreme data compression [5]. This means that AvatarStudio uses the most important aspects of the 3D shapes, and thus has "learned" the most important aspects of what human geometry looks like. In this particular use case, 100 main components are used $[6,7,8]$. Since each major component has about $500 \mathrm{k} 3 \mathrm{D}$ vectors $[9,10]$, this gives us the possibility to use a relatively compact representation of the most important aspects of the 3D data observed in the serial measurement. The data structure used is about $0.5 \mathrm{~GB}$ for each gender. This means that it can be kept completely in the computer memory during runtime, resulting in a very fast and interactive mechanism of avatar generation ( 20 ms per avatar generation).

Another positive side effect of the compression aspect is that the anonymity of the output can always be guaranteed, which is very important nowadays. Using PCA technology, the output range is not necessarily limited to the observed shapes, which fills the "holes" between the observations and also allows meaningful output to regions outside the observation range, e.g. for more extreme body types [11,12].

Of course, the PCA is only the basic component of the AvatarStudio technology. The technology also uses other (statistical and mathematical) approaches to calculate the precise linear combination of eigenvalues to produce a specific, valid and accurate avatar output. Fig. 2 shows avatars for DOB 40 and HAKA 106 size, defined on 22 typical measurements of an industry size chart.
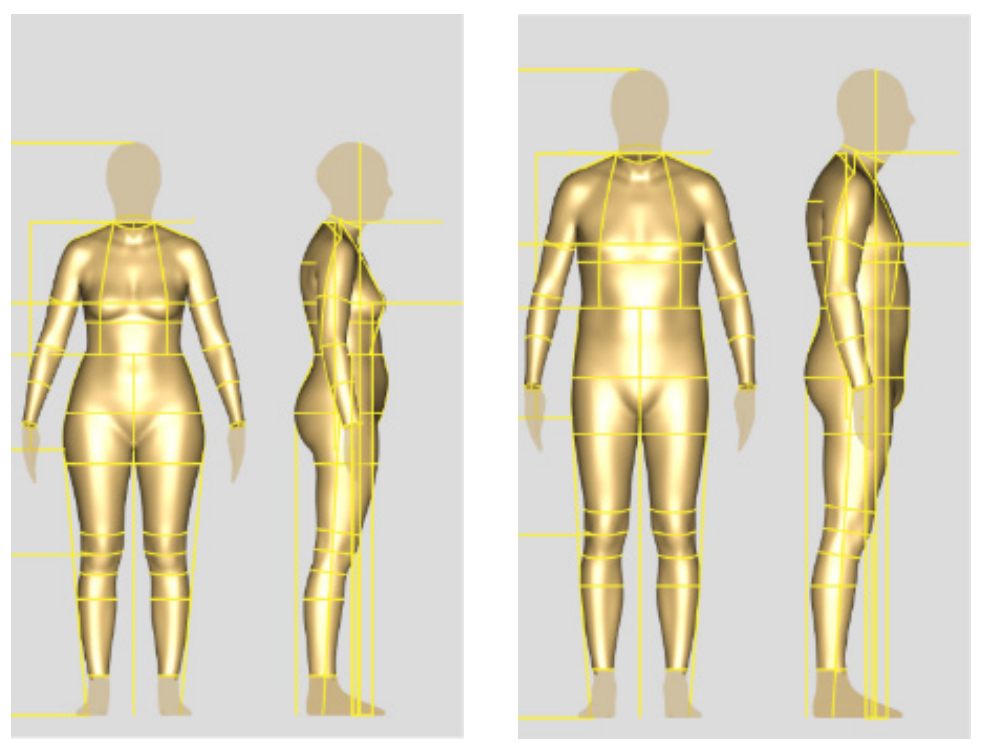

Figure 2 Female and male avatar based on 22 measurements

\section{Avatar Concept}

The design of the specific MtM avatar control dimensions, or more precisely the selection of the so-called features or feature vectors (generic term for the sum of parameters defining an avatar) is deliberately based on typical design rules as in mechanical or civil engineering (technical drawings).

Accordingly, perimeter measurements are provided with a height component. The specific portion of each such segment can be configured more precisely through its width and depth. Equipped with all these input 
parameters, a wide range of body types and morphological aspects can be expressed and systematically investigated (Fig. 3).

Fig. 4 shows two avatars generated from exactly the same body dimensions (HAKA 106S), with the morphological variation of triangle and inverted triangle.
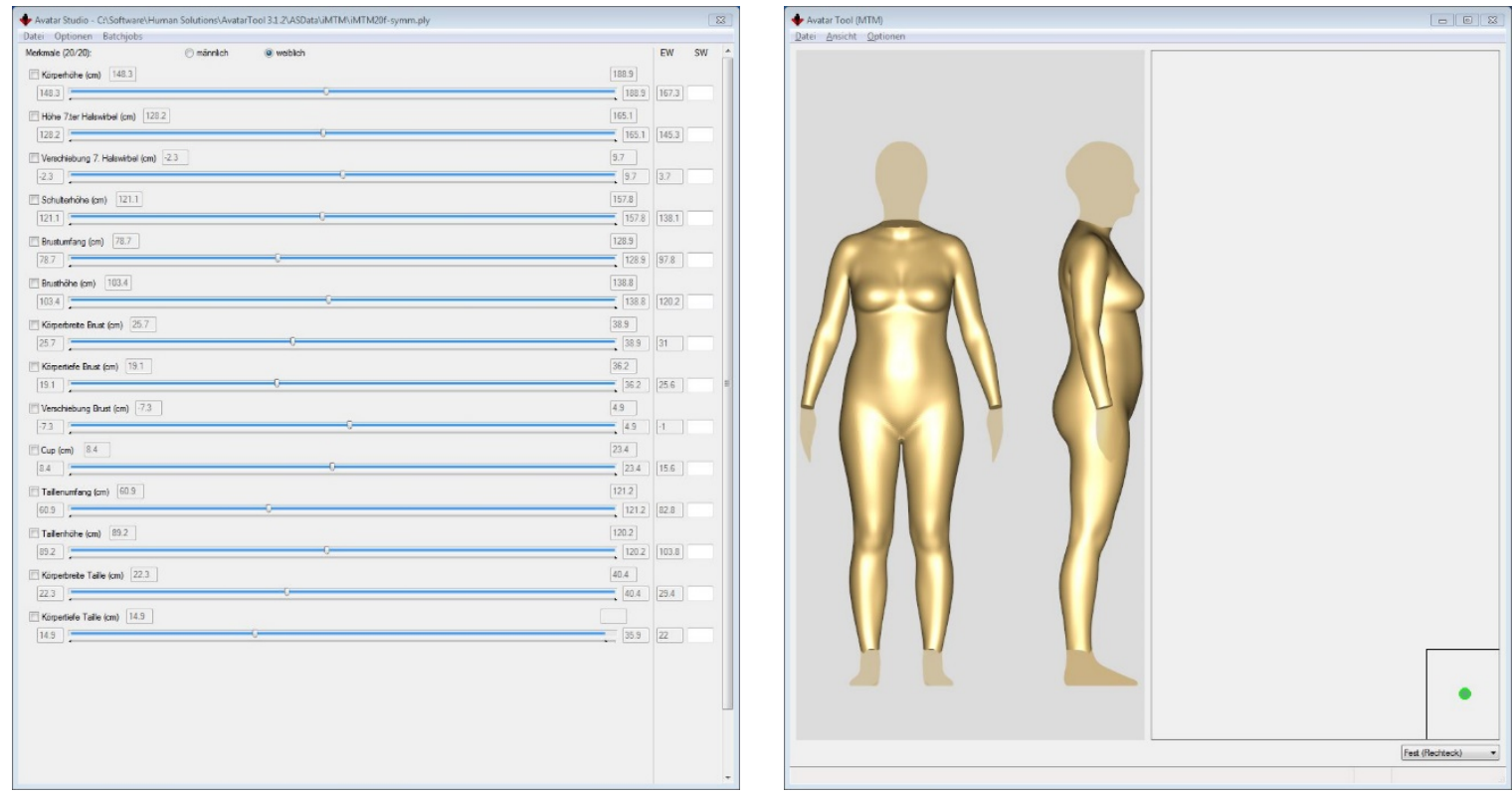

Figure 3 AvatarTool
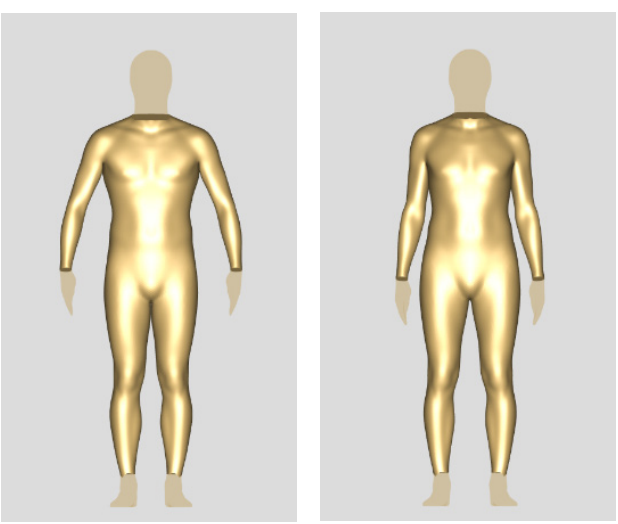

Figure 4 Male avatars with same body measurements but different morphological shape

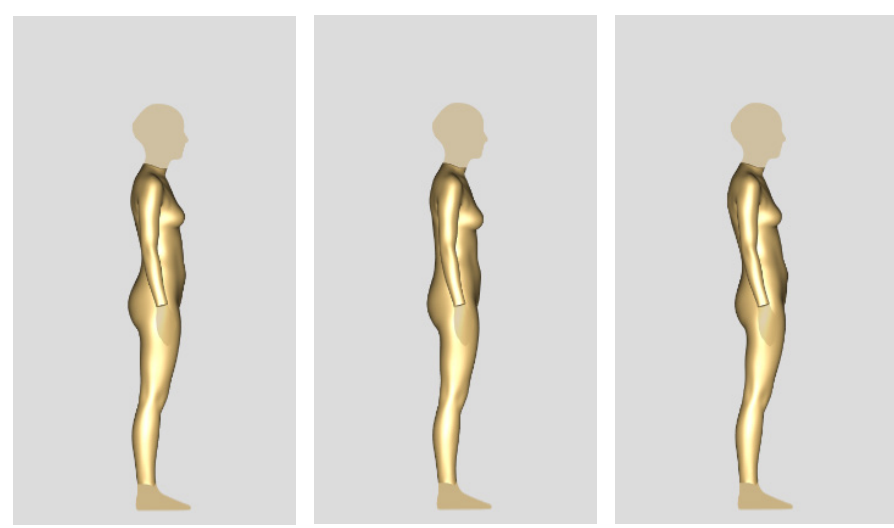

Figure 5 Female avatars vary in shape and posture

In addition to that, special features had been introduced that control typical postural aspects to e.g. examine to which extend a specific body (or body size) given by its measurements may still vary in its shape and posture (Fig. 5), and to what extend this affects the fit.

\subsection{Automatic evaluation of MtM values based on an avatar test population}

Using garment simulation on avatar test populations provides an opportunity to validate the MtM grading process and, by visualizing the simulation results, enable the user to approve size, fit and design.

Automatic creation of an avatar test population with automatic measurement creation and output for MtM From an architectural point of view, the system consists of 3 major components:

1. the avatar test population definition system (AvatarTool)

2. a batch executing system including (BatchSimulator)

- avatar measuring 
- size recommendation and calculation of alteration values

- a garment simulation (Vidya) system in combination with a CAD system including MtM functionality 3. result viewing and simulation comparison system (SimulationViewer)

\subsection{Research methodology}

Investigations were carried out with various MtM products of classical women's and men's clothing as well as workwear. All products have been fitted and evaluated on the avatar test population with 3D garment simulation applications - Vidya, V-Stitcher - regarding realistic material simulation following the research methodology shown in Fig. 6.

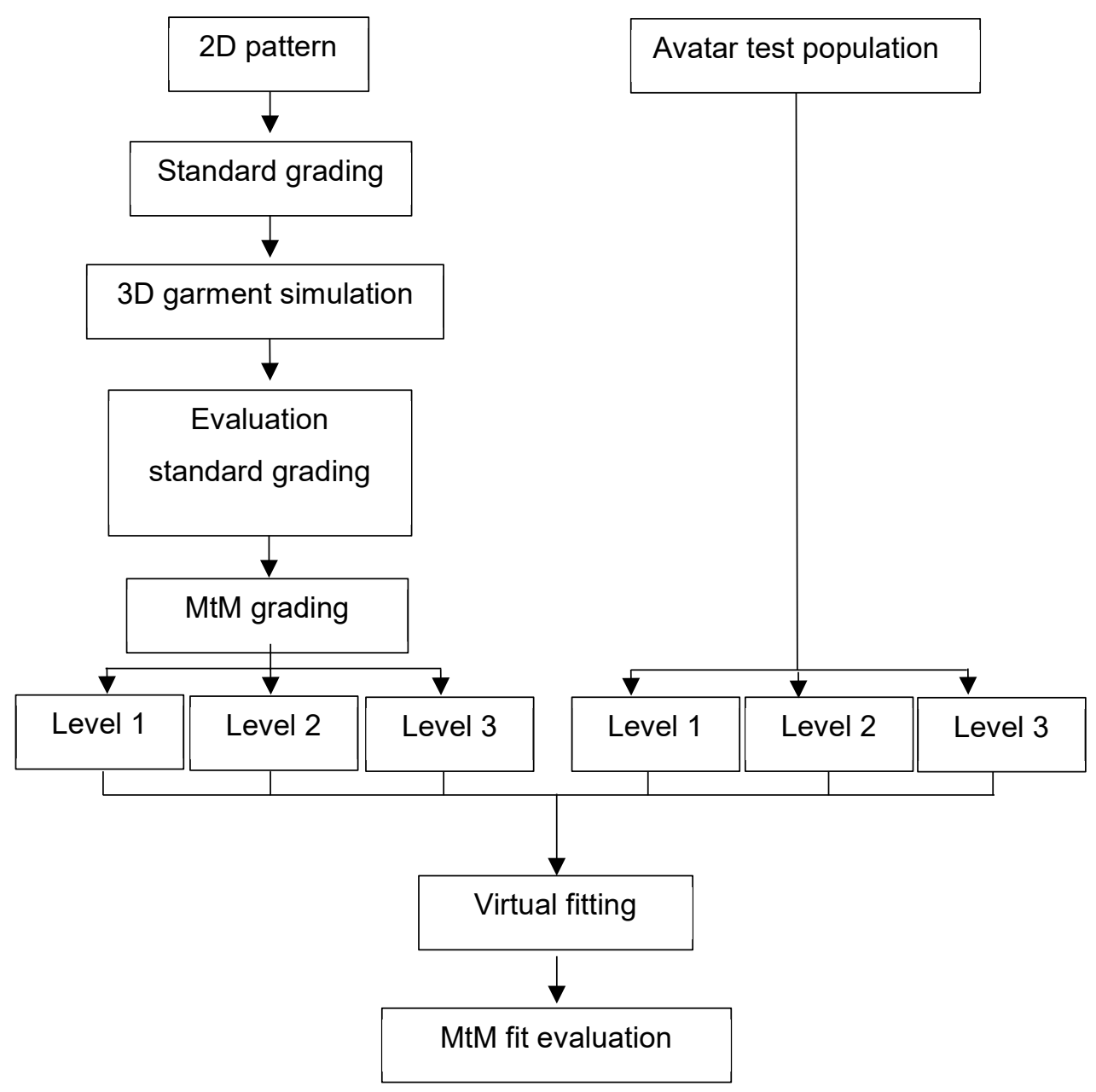

Figure 6 Research methodology

\section{$2.4 \quad$ Target group}

The target group for MtM garments is related to the target group of the defined standard sizes. The aim is to dress customers with bodies and shapes different from the standard sizes.

\section{Definition of target group}

The target group investigated comprises female and male avatars split into three groups, depending on the predefined MtM modifications:

- Level 1: Length modifications

- Level 2: Length and girth modifications + distribution of girth modifications

- Level 3: Posture modifications 
All test avatars based on statistically verified bodies and therefore extreme body data are excluded and not part of the survey. Figure 7 shows a selection of the target group.

\section{Size recommendation}

The selection of the product-related reference size is based on chest, waist and hip circumference for outerwear, and only waist and hip circumference for lower body garments. For this purpose the measurements are evaluated with different priorities.

\section{Calculation of alteration values}

Alteration values are calculated by the difference of individual measurements to the values in the size chart.
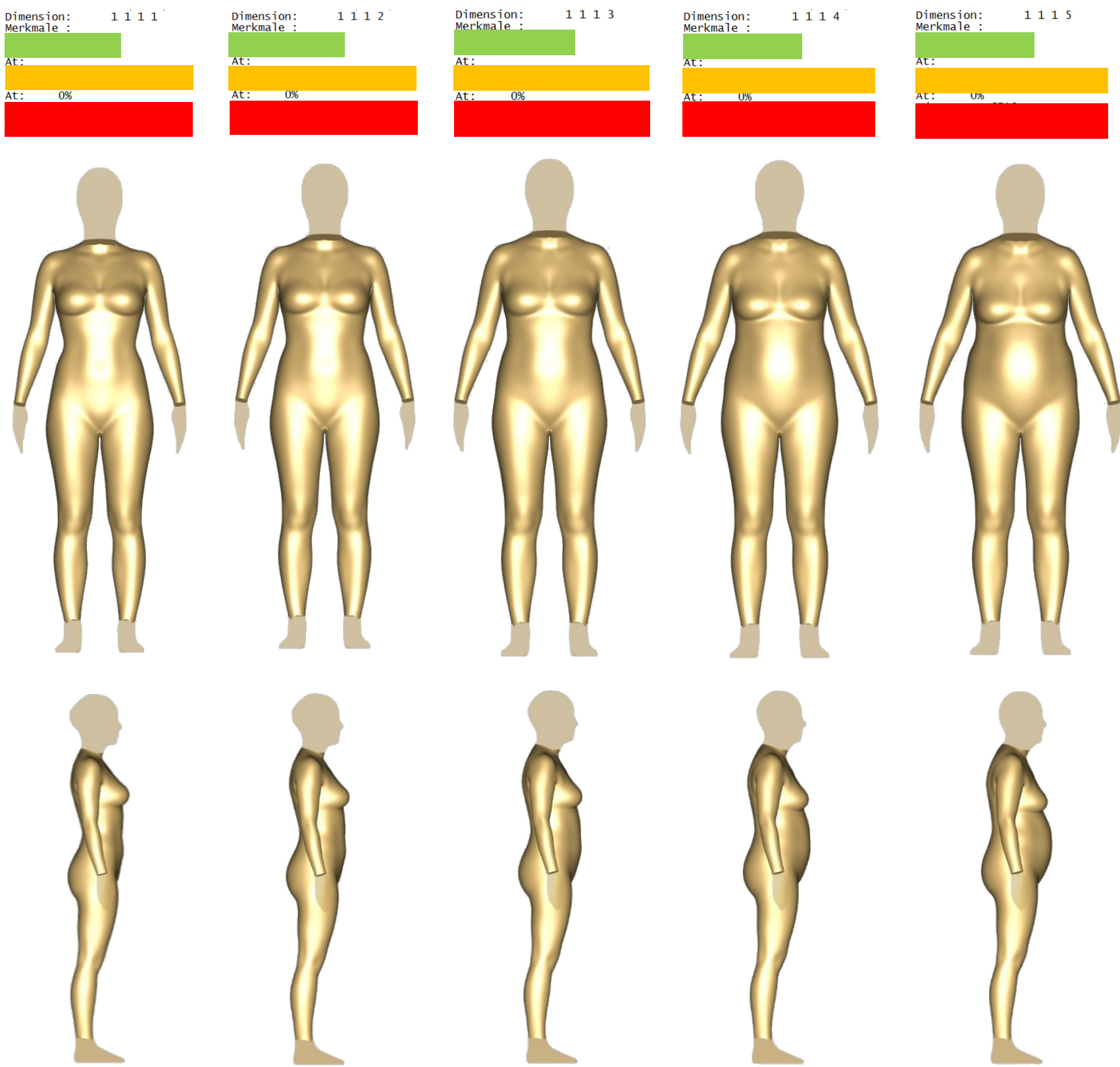

Figure 7 Target group with same body height and hip girth but different waist girths 
Divided into four areas - length modifications, girth modifications, distribution of girth measurements and posture - rules are developed to transfer the modifications directly to the MtM grading process. The overview below shows the different rules for each area.

- Length modifications: difference of individual and standard body measurement

- Girth modifications: difference of individual and standard body measurement, with standard distribution based on the selected construction system

- Distribution of girth measurements: the distribution is measured on the individual body and the difference to the standard distribution is used for the MtM rule

- Posture: comparison of different body measurements, e.g. for a hollow back comparison of neck to waist center back with neck to waist over bust

\section{Product group}

Representative for the investigated products - classic products of skirts, blouses, shirts and trousers as well as workwear products -, the results of a blouse and a smock overall are shown below.

- CAD pattern: The product development as well as the standard and MtM grading is done for all products with the 2D CAD system Cad.Assyst. A second test with the 2D CAD system Grafis has shown that the process can be transferred to each other 2D CAD system.

- Basic pattern: Both patterns - blouse and smock overall - based on the iSize body measurement chart for women from size 32 to 60.

- MtM Grading: Based on the reference size, the predefined MtM-values will be automatically calculated and directly imported to the CAD System.

\subsection{D Simulation}

3D simulations are used to evaluate the fit at an early stage - before the start of a manufacturing process. Virtual stitching is performed with the Vidya and V-Stitcher 3D CAD systems to ensure that a virtual fit is not dependent on a specific 3D CAD system.

\section{MtM fitting}

The MtM fit will be checked for the avatar test population and different products using a batch mode of the 3D simulation system Vidya. The output of different simulation pictures for each single simulation offers the possibility to verify the MtM fit.

\section{Fit evaluation}

Figure 8 gives a review about the fit evaluation based on virtual try-ons. Different simulation views like body-distance, elongation, and elasticity give detailed information about the fitting. 


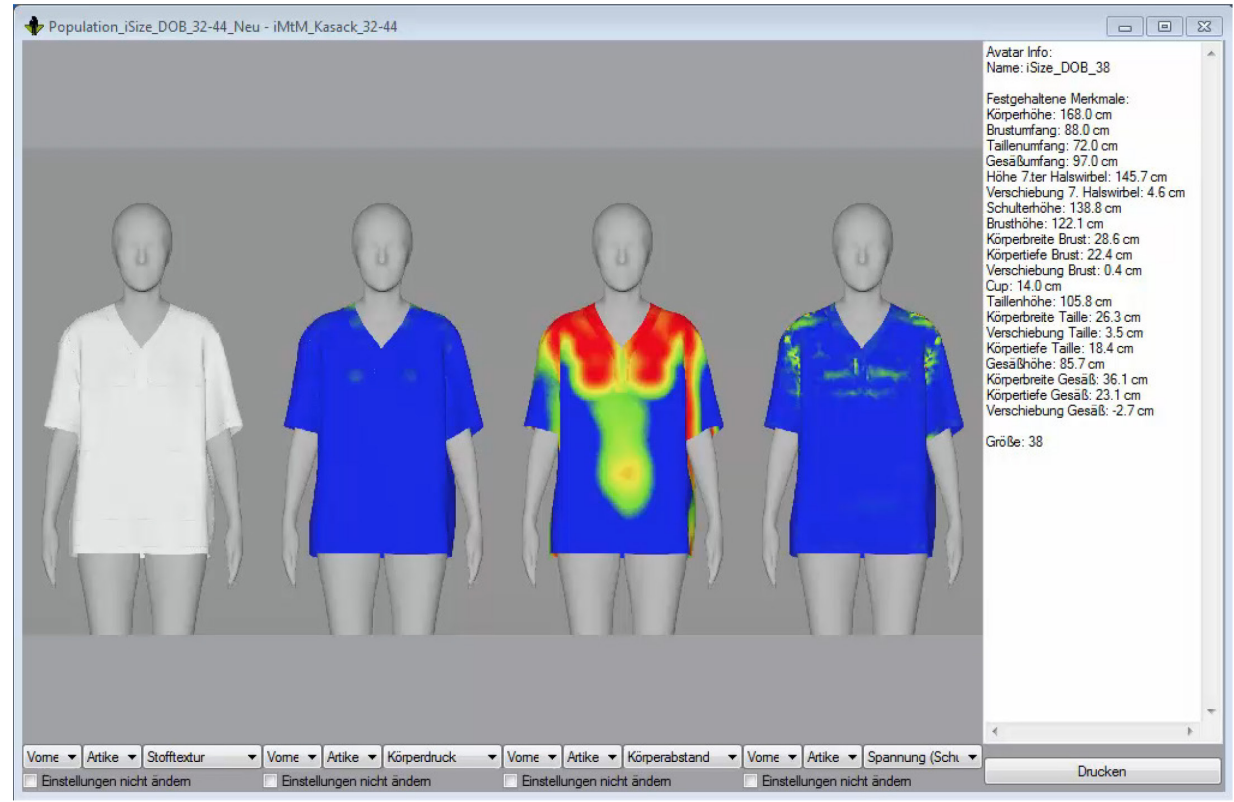

Figure 8 Simulation Viewer - simulation results for fit control

\section{Results}

The following results (Figs. 9, 10) are just a few samples from tests conducted with a large Avatar test population of both men and women and the resulting adjustments. They are divided into four sections: the generation of the avatar test population, the validation of the measurements of the avatar test population obtained with a special software application, the approach of implementing posture and shape parameters into the MtM pattern design, and the 3D fit studies for accelerated preliminary fit testing within the MtM process.
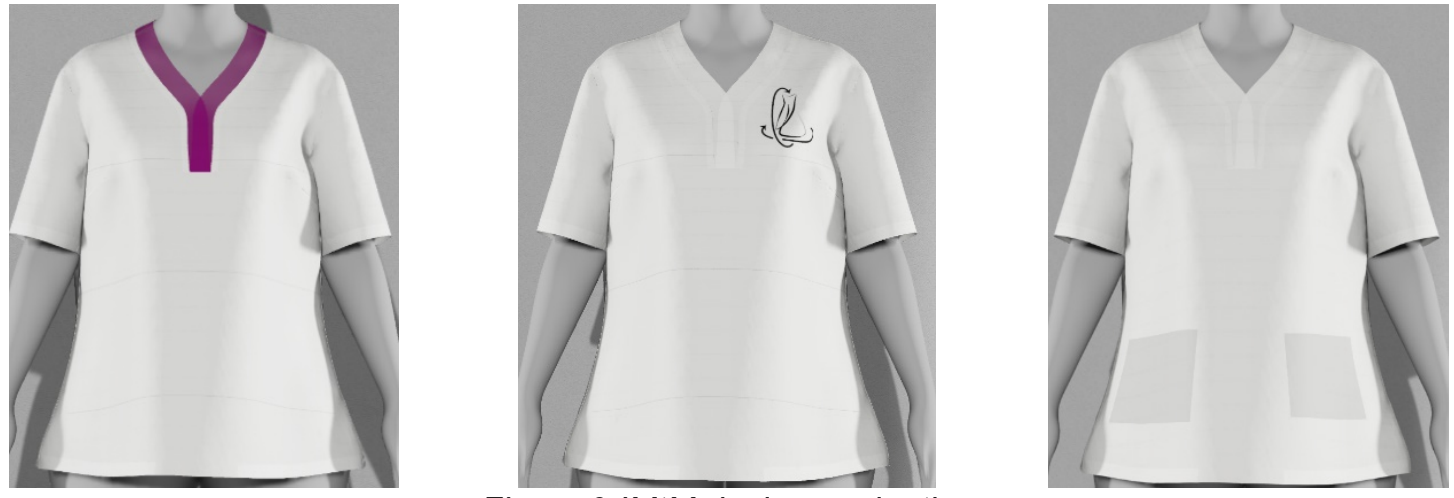

Figure 9 IMtM design evaluation

\begin{tabular}{|l|c|c|c|}
\hline & Reference size & MtM grading & Body distance \\
\hline & &
\end{tabular}




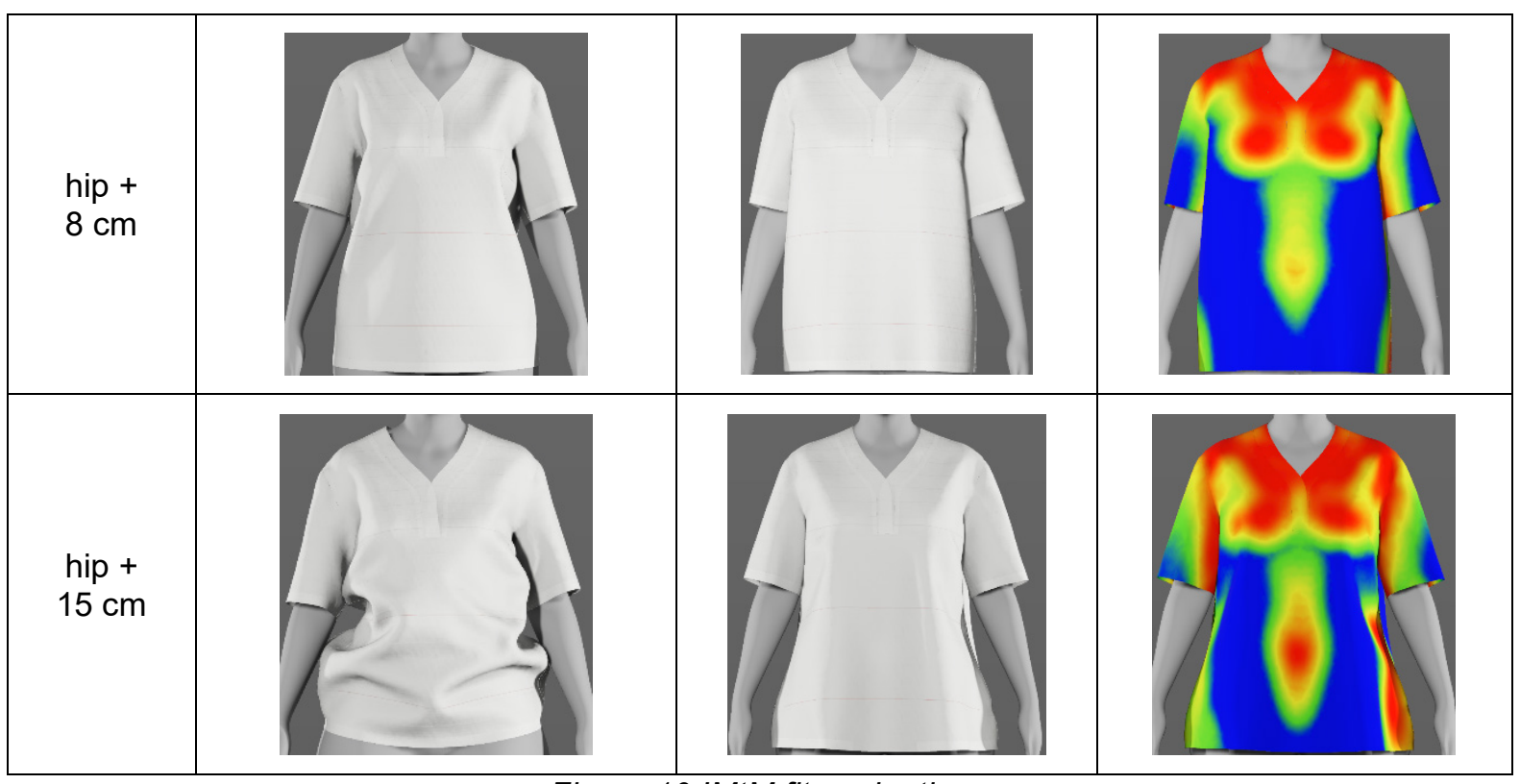

Figure 10 IMtM fit evaluation

\section{Future Works}

This work presents new software solutions with an interface to the simulation software Vidya. As Avalution and Assyst belong to the Human Solutions Group, the development of an interface to their own software has proven to be useful. Alternative developments with different 3D-software are conceivable and can be subject of separate investigation. 


\section{Conclusion}

The findings of this paper have demonstrated the importance and usefulness of examining MtM classification at an early stage of the product development process. The automatic creation of avatar test populations and the batch mode for the 3D simulations are very helpful tools to make the virtual try-ons fast and available for the MtM validation process. Knowing that not all individual topics can be covered by the MtM process, a realistic avatar test population can be visualized for industrial applications, and the extraction of measurements provides multiple MtM information. The algorithm for evaluating the size recommendation is very flexible and can be specified for individual purposes.

The next step to complete the whole process aims to simplify the fit validation step by providing an automated and objective evaluation system for the apparel industry.

\section{Acknowledgment}

This work was performed within the cooperative Project ZIM ZF4102205CJ and the authors would like to thank to BMWi -AIF Projekt GmbH for the financial support of this investigation.

\section{References}

[1] Ernst, M.; Detering-Koll, U. Posture Dependency of 3D-Body Scanning Data for a Virtual Product Development Process. In Proc. of 5th International Conference on 3D Body Scanning Technologies, Lugano, Switzerland, 21 22 October 2014.

[2] Löffler-Wirth, H. Body typing of the adult population of Leipzig. Application forum Human Solutions. Kaiserslautern, Germany, 21 April 2016.

[3] Vidya, Assyst. Available online: https://www.assyst.de/de/produkte/3d-vidya/index.html (accessed on February 19, 2020)

[4] SizeGERMANY - Startseite'. Available online: https://portal.sizegermany.de/SizeGermany/pages/home.seam (accessed on February 19, 2020).

[5] Fischer, T.; Artschwager, A.; Pfleiderer, K.; Rissiek, A.; Mandalka, M.; Seidl, A.; Trieb, R. Automatic morphological classification with Case-Based Reasoning. 7th International Conference on 3D Body Scanning Technologies. Lugano, Switzerland, 30 Nov. - 1 Dec. 2016.

[6] Ernst, M.; Detering-Koll, U. Investigation on Body shaping Structures Using Body Scanning Technology and 3D Simulation Tools. In Proc. of 3nd International Conference on 3D Body Scanning Technologies, Lugano, Switzerland, 16-17 October 2012.

[7] Ernst, M. Next generation of product development - obtaining 3D buy-in, building capability and generating a fruitful roadmap. PI Conference Berlin, Germany, 5-6 October 2016.

[8] Ernst, M.; Böhm, M.; Detering-Koll, U. Rapid body scanning technology for a virtual mass customization process in garment industry. In Proc. of 7th International Conference on 3D Body Scanning Technologies, Lugano, Switzerland, 30 Nov. - 1 Dec. 2016.

[9] Allen, B.; Curless, B.; Popovic, Z. The space of human body shapes: reconstruction and parameterization from range scans. In Proc. of SIGGRAPH '03 ACM SIGGRAPH 2003 Papers, 587-594, San Diego, California: ACM New York.

[10] Blanz, V.; Vetter, T. A morphable model for the synthesis of 3D faces. In Proceedings of ACM SIGGRAPH 99, 187-194, 1999.

[11] Praun, E.; Sweldens, W.; Schröder, P. Consistent mesh parameterizations. In Proceedings of ACM SIGGRAPH 2001, 179-184. New York: ACM Press/ACMSIGGRAPH.

[12] Turk, M.; Pentland, A. Eigenfaces for recognition. Journal of Cognitive Neuroscience, 1991, 3 (1), 71-86. DOI: 10.1162/jocn.1991.3.1.71. 